\title{
Streptococcus anginosus Empyema Induced by Dental Abscess: A Case Report
}

\author{
Ishan Patel ${ }^{\mathrm{a}}$, Steven Douedia, e, Shraddhadevi Makadia ${ }^{\mathrm{b}}$, Vandan Upadhyaya ${ }^{\mathrm{a}}$, \\ Arda Akoluk ${ }^{\mathrm{a}}$, Jennifer Douedic, Mohammad Hossain ${ }^{\mathrm{a}}$, Edward Liu ${ }^{\mathrm{d}}$
}

\begin{abstract}
Streptococcus anginosus is a gram-positive catalase-negative cocci and commensal flora of the oropharynx and gastrointestinal tract. Streptococcus anginosus is known to be highly virulent and known to cause invasive pyogenic infection which may necessitate emergent surgical treatment. We present a case of a 53-year-old female with dental caries and uncontrolled diabetes mellitus who presented with progressive shortness of breath for 1 week. A computed tomography scan of the chest showed a loculated empyema which was treated with emergent video-assisted thoracoscopic surgery. Pleural fluid culture as well as sputum cultures grew Streptococcus anginosus. The patient was treated intravenous cephalosporins and discharged on oral antibiotics after 14 days with outpatient dental follow-up and improvement of symptoms.
\end{abstract}

Keywords: Streptococcus anginosus; Empyema; Dental caries; Diabetes mellitus; VATS

\section{Introduction}

Of the 1 million patients hospitalized in USA with pneumonia, $20-40 \%$ of them have a parapneumonic effusion and $5-10 \%$ of these parapneumonic effusions progress to empyema [1]. Pathogens associated with community acquired parapneumonic effusion and empyema include Streptococcus pneumoniae (S. pneumoniae), Streptococcus anginosus (S. anginosus)

Manuscript submitted July 14, 2020, accepted July 21, 2020

Published online August 6, 2020

aDepartment of Medicine, Jersey Shore University Medical Center, Hackensack Meridian Health, Neptune, NJ 07753, USA

${ }^{b}$ Department of Medicine, Ocala Regional Medical Center, University of Central Florida, Ocala, FL 07753, USA

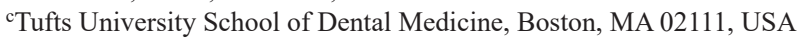

${ }^{\mathrm{d}}$ Department of Infectious Disease, Jersey Shore University Medical Center, Hackensack Meridian Health, Neptune, NJ 07753, USA

${ }^{e}$ Corresponding Author: Steven Douedi, Department of Medicine, Hackensack Meridian Health, Jersey Shore University Medical Center, 1945 Route 33, Neptune, NJ 07753, USA. Email: Steven.Douedi@hackensackmeridian.org

doi: https://doi.org/10.14740/jmc3547 group and anaerobes (Fusobacterium species, Prevotella species, Peptostreptococcus species, and Bacteroides species). Pathogens associated with hospital acquired parapneumonic effusion and empyema include Staphylococcus aureus, Escherichia coli, Pseudomonas aeruginosa, and Klebsiella pneumoniae [1]. S. anginosus group forms commensal flora of oral cavity, upper respiratory tract, gastrointestinal tract and genitourinary tract, and is known for causing parapneumonic effusion and empyema [2,3]. S. anginosus group can become pathogenic and cause contiguous or distant infections after mucosal damage causing [4]. Empyema thoracis is defined as collection of pus in the pleural space and has been associated with high mortality rate which ranges between $6 \%$ and $24 \%$ [5]. Treatments of this condition include medical management with antibiotics and complete drainage of the infected fluid by various methods like chest tube placement, video-assisted thoracoscopic surgery (VATS), or open thoracotomy. Early surgical management is warranted when there is radiographic evidence of loculations as these are predictors of failure of treatment with increased mortality [6]. Here we present a case of loculated empyema likely developed from untreated dental caries initially treated with antibiotics and chest tube placement followed by VATS with decortication surgery.

\section{Case Report}

A 53-year-old female with medical history of uncontrolled diabetes mellitus (most recent hemoglobin A1c 14.5\%) presented to the emergency department with complaints of shortness of breath and left sided back pain for 1 week. Her shortness of breath became rapidly progressive with worsening on minimal ambulation prompting her to come to the emergency department. She denied any fever, cough, or sputum production on admission. On review of systems, she also complains of left sided tooth pain that has been present for several months of which she did not seek treatment. Vitals on admission were: blood pressure of 141/96 $\mathrm{mm} \mathrm{Hg}$, heart rate of 116 beats $/ \mathrm{min}$, respiratory rate of $26 / \mathrm{min}$, temperature of $37.0^{\circ} \mathrm{C}$, and oxygen saturation of $98 \%$ on 4-L nasal cannula. Oral examination revealed mandibular left first molar tooth decay with fracture and caries with abscess forming in the buccal vestibule of which cultures were obtained and found to grow S. anginosus.

Respiratory examination revealed tracheal shift to right side with absence of breath sounds on left side and dullness 


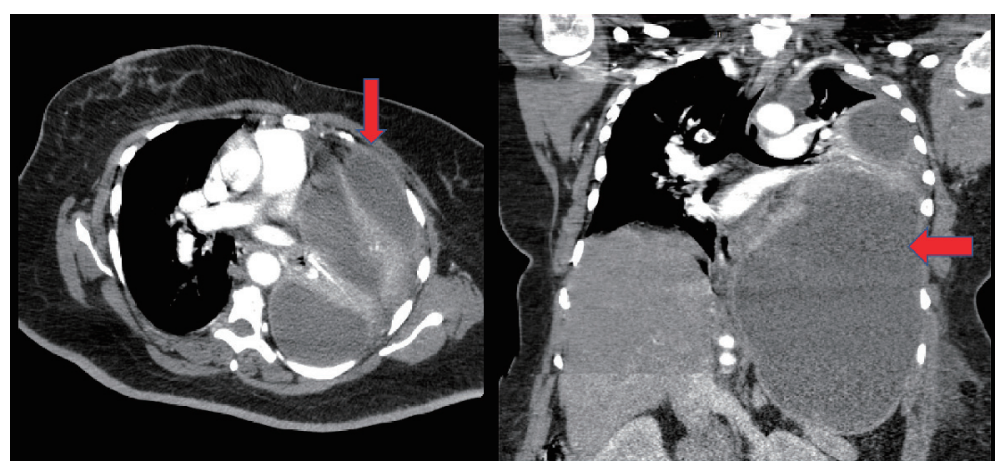

Figure 1. CT scan of the chest, abdomen, and pelvis revealing a large left sided loculated effusion with foci of gas concerning for empyema (red arrow) along with compressive atelectasis of most of the left upper and lower lobes and mediastinal shift to right. CT: computed tomography.

on percussion. Complete blood count showed a white blood cell count of $18 \times 10^{3} / \mu \mathrm{L}$ (normal range: $4.5-11.0 \times 10^{3} / \mu \mathrm{L}$ ), neutrophils of $77 \%$ (normal range: $50-70 \%$ ), thrombocytosis with a platelet count of $643 \times 10^{3} / \mu \mathrm{L}$ (normal range: 140 $450 \times 10^{3} / \mu \mathrm{L}$ ), anion gap of 23 , and metabolic acidosis due to diabetic ketoacidosis (DKA) with a serum bicarbonate of 14, blood sugar of $515 \mathrm{mg} / \mathrm{dL}$ (normal range: 70 - $99 \mathrm{mg} / \mathrm{dL}$ ), beta-hydroxybutyrate of $>8 \mathrm{mmol} / \mathrm{L}$ (normal range: 0.02 $0.27 \mathrm{mmol} / \mathrm{L}$ ), lactic acid of $1.2 \mathrm{mmol} / \mathrm{L}$ (normal range: 0.5 - $2.0 \mathrm{mmol} / \mathrm{L}$ ) and venous blood gas revealing a $\mathrm{pH}$ of 7.18, $\mathrm{CO}_{2}$ of $13.3 \mathrm{~mm} \mathrm{Hg}, \mathrm{O}_{2}$ of $57 \mathrm{~mm} \mathrm{Hg}$, and bicarbonate level of $4.8 \mathrm{mmol} / \mathrm{L}$ (normal range: 22 - $26 \mathrm{mmol} / \mathrm{L}$ ). A computed tomography (CT) scan of chest was negative for pulmonary embolism but showed large left sided loculated effusion with foci of gas concerning for empyema along with compressive atelectasis of most of the left upper and lower lobes and mediastinal shift to right (Fig. 1).

Due to loculated empyema the patient had emergent chest tube placement which drained $5,000 \mathrm{~mL}$ of pus. The patient was empirically started on piperacillin-tazobactam and was aggressively treated with intravenous fluids and insulin drip due to DKA and was transferred to the intensive care unit for further management. Pleural fluid studies showed a white blood cell count of 370,240 (normal range: 1,000 - 5,000 cells $/ \mathrm{mm}^{3}$ ) with $44 \%$ neutrophils, lactic acid dehydrogenase of 2,075 (normal value $<50 \%$ plasma level), and glucose level of 10 (normal value should be about plasma level, $<40$ indicative of empyema) consistent with exudative effusion. Patient had a repeat CT scan of chest which showed interval improvement but presence of residual collection roughly measuring $300 \mathrm{~mL}$ and dense left lower lobe consolidation which was unmasked after drainage.

On day 2, Gram staining of the pleural fluid and sputum cultures grew Gram-positive cocci in chains and the patient underwent VATS with decortication and second chest tube placement with an additional $300 \mathrm{~mL}$ of pus being removed. Post procedure the patient had to be intubated due to worsening respiratory distress. On day 3 the patient was successfully extubated. Pleural fluid and sputum cultures confirmed growth of $S$. anginosus. The patient's DKA eventually resolved and she was transitioned to basal bolus insulin regimen. As per sensitivity antibiotics were downgraded to ampicillin-sulbactam. On day 4 her antibiotics were switched to amoxicillin-clavulanic acid and the patient was transferred to general medical floors for further management. Monitoring of empyema was done with serial chest X-rays and she had removal of chest tubes on day 9 and day 11 of hospitalization. On day 14 she was discharged with oral amoxicillin-clavulanic acid for an additional 4 weeks. She ultimately followed up with dentist outpatient and underwent removal of the infected dental caries and management of her oral abscess. On follow-up 1 month after discharge, the patient remained asymptomatic and in good health.

\section{Discussion}

The Streptococcus milleri ( $S$. milleri) group is comprised of $S$. anginosus, Streptococcus constellatus (S. constellatus) and Streptococcus intermedius (S. intermedius), which are grampositive catalase-negative organisms commonly found in the upper respiratory, digestive and reproductive tracts [3, 7]. $S$. anginosus was first described in 1956 causing a dental abscess but has also been found to be the cause of empyema and other thoracic infections $[3,8,9]$. Known for being highly virulent, thoracic infections associated with $S$. anginosus have been associated with a mortality rate of up to $15-30 \%[8,10]$. For this reason, early and aggressive management with antibiotics and surgical intervention has been recommended especially when an empyema is identified [11].

We presented a case of a middle-aged female who had poor oral hygiene and was found to have a dental abscess colonized with $S$. anginosus. While initially presenting with only shortness of breath, she was found to have a severe loculated empyema due to $S$. anginosus infection. Antimicrobial management of $S$. anginosus has shown to be relatively simple as the $S$. milleri group was all found to be susceptible to penicillin, ampicillin, and ceftriaxone [12]. For this reason, our patient's antibiotics were decelerated to ampicillin-sulbactam after sensitivities confirmed susceptibility. The mean treatment length for $S$. milleri group has been about 34 days; however, the decision was made to treat our patient for a total of 6 weeks [13].

In addition to early antibiotic management, early surgical intervention leads to favorable outcomes in our patient. In retrospective studies, $S$. anginosus was found to cause severe em- 
pyema and removal of purulent drainage, as with a chest tube, improved patient survival [11]. In addition, loculated or purulent empyema has been defined as an indication for surgical intervention using VATS $[13,14]$. As described in our case, the patient presented developed a large loculated empyema due to $S$. anginosus infection. The empyema was ultimately managed with two chest tubes each expelling significant drainage and ultimately VATS.

\section{Conclusions}

$S$. anginosus, a member of the $S$. milleri group, possesses a high virulence and when causing a thoracic infection can lead to a significantly elevated mortality. Treatment guidelines have been developed for this dangerous infection and included antibiotics, drainage of purulent material, and early surgical intervention as done in our case. Prompt recognition and management of $S$. anginosus infections can lead to favorable outcomes.

\section{Acknowledgments}

None to declare.

\section{Financial Disclosure}

This research did not receive any specific grant from funding agencies in the public, commercial, or not-for-profit sectors.

\section{Conflict of Interest}

The authors declare that there is no conflict of interest regarding the publication of this paper.

\section{Informed Consent}

The patient described has given his informed consent to publish this manuscript.

\section{Author Contributions}

IP, SD, SM, VU, AA, and JD contributed to visualization and writing (original draft, review, and editing). MH and EL contributed to writing (review and editing).

\section{Data Availability}

The authors declare that data supporting the findings of this study are available within the article.

\section{References}

1. Light RW. Parapneumonic effusions and empyema. Proc Am Thorac Soc. 2006;3(1):75-80.

2. Wong CA, Donald F, Macfarlane JT. Streptococcus milleri pulmonary disease: a review and clinical description of 25 patients. Thorax. 1995;50(10):1093-1096.

3. Jiang S, Li M, Fu T, Shan F, Jiang L, Shao Z. Clinical Characteristics of Infections Caused by Streptococcus Anginosus Group. Sci Rep. 2020;10(1):9032.

4. Piscitelli SC, Shwed J, Schreckenberger P, Danziger LH. Streptococcus milleri group: renewed interest in an elusive pathogen. Eur J Clin Microbiol Infect Dis. 1992;11(6):491-498.

5. Ahmed AE, Yacoub TE. Empyema thoracis. Clin Med Insights Circ Respir Pulm Med. 2010;4:1-8.

6. Semenkovich TR, Olsen MA, Puri V, Meyers BF, Kozower BD. Current state of empyema management. Ann Thorac Surg. 2018;105(6):1589-1596.

7. Whiley RA, Beighton D, Winstanley TG, Fraser HY, Hardie JM. Streptococcus intermedius, Streptococcus constellatus, and Streptococcus anginosus (the Streptococcus milleri group): association with different body sites and clinical infections. J Clin Microbiol. 1992;30(1):243-244.

8. Sunwoo BY, Miller WT, Jr. Streptococcus anginosus infections: crossing tissue planes. Chest. 2014;146(4):e121e125.

9. Porta G, Rodriguez-Carballeira M, Gomez L, Salavert M, Freixas N, Xercavins M, Garau J. Thoracic infection caused by Streptococcus milleri. Eur Respir J. 1998;12(2):357-362.

10. Petti CA, Stratton IV, Mandell GL, Bennett JE, Dolin R. Streptococcus anginosus group. In: Mandell, Douglas, and Bennett's principles and practice of infectious diseases 7th ed. Philadelphia, PA: Churchill Livingstone Elsevier. 2010;2681-2685.

11. Stelzmueller I, Biebl M, Berger N, Eller M, Mendez J, Fille M, Angerer K, et al. Relevance of group Milleri streptococci in thoracic surgery: a clinical update. Am Surg. 2007;73(5):492-497.

12. Tracy M, Wanahita A, Shuhatovich Y, Goldsmith EA, Clarridge JE, 3rd, Musher DM. Antibiotic susceptibilities of genetically characterized Streptococcus milleri group strains. Antimicrob Agents Chemother. 2001;45(5):15111514.

13. Reis-Melo A, Soares D, Magalhaes MF, Ferraz C, Vaz L. Complicated pneumonia with empyema caused by streptococcus Anginosus in a child. Rev Paul Pediatr. 2020;38:e2018258.

14. Nose N, Anami T. Simultaneous bilateral decortications via video-assisted thoracic surgery for bilateral empyema. Int J Surg Case Rep. 2015;6C:81-83. 